\title{
ON THE SPECTRAL MULTIPLICITY OF A CLASS OF FINITE RANK TRANSFORMATIONS
}

\author{
G. R. GOODSON
}

\begin{abstract}
The rank $M$ transformations, which Chacon called the simple approximations with multiplicity $M$, were shown by Chacon to have maximal spectral multiplicity at most $M$, although no example was given where this bound is attained for $M>1$. In this paper, for each natural number $M>1$, we show how to construct a simple approximation with multiplicity $M$ which is ergodic and has maximal spectral multiplicity equal to $M-1$.
\end{abstract}

Introduction. The purpose of this paper is to give a method of constructing ergodic automorphisms $\tau:[0,1) \rightarrow[0,1)$ with finite rank and maximal spectral multiplicity greater than one. In particular for each natural number $M>1$ we show how to construct ergodic automorphisms which admit simple approximations with multiplicity $M$ (in the sense of Chacon [2]) and have maximal spectral multiplicity equal to $M-1$.

Oseledec [5], using interval exchange transformations, was the first to construct an ergodic automorphism with finite spectral multiplicity greater than one. His example involved an exchange of 30 intervals and had maximal spectral multiplicity lying between 2 and 30 . Robinson [7] and Katok (unpublished) generalised his result again using interval exchange transformations. Katok constructed an example with multiplicity equal to 2 and Robinson constructed ergodic automorphisms with arbitrary finite maximal spectral multiplicity. Our examples are constructed using the stacking method with $M$-columns; they are ergodic having discrete spectrum on an invariant subspace and continuous spectrum with multiplicity $M-1$ on its orthogonal complement. The main significance of the construction is its simplicity. The construction is based on an example of del Junco [3] and Baxter [1].

1. Preliminaries. Let $\tau:(X, \mathfrak{B}, \mu) \rightarrow(X, \mathfrak{B}, \mu)$ be an invertible measure preserving transformation of a Lebesgue space $(X, \mathfrak{B}, \mu)$, and let $U_{\tau}: L^{2}(X) \rightarrow L^{2}(X)$ be the induced unitary operator defined by $\left(U_{\tau} f\right)(x)=f\left(\tau^{-1} x\right)$. Then the maximal spectral multiplicity of $U_{\tau}$ is defined as follows.

DEFINITION 1. Let $U: H \rightarrow H$ be a unitary operator on a Hilbert space $H$. The maximal spectral multiplicity of $U$ is $m(U)=\inf \left\{m \in \mathbf{Z}^{+} \cup\{\infty\}: \exists f_{1}, \ldots, f_{m} \in H\right.$ such that linear combinations of $U^{i} f_{j}, i \in \mathbf{Z}, j=1, \ldots, m$, are dense in $\left.H\right\}$.

When the multiplicity is one we say the spectrum is simple. If, for $U_{\tau}$, the eigenvalue 1 is simple and is the only eigenvalue, we say $\tau$ has continuous spectrum. (See Robinson [7] and Parry [6] for more details and a discussion of the history of the spectral theory of measure preserving transformations.)

DEFINITION 2. A partition of $X$ is a finite collection $\zeta=\left\{A_{i}: i \in I, A_{i} \in \mathfrak{B}\right\}$ of disjoint sets. A $\tau$-stack $S$ is a partition $\left\{S_{0}, \ldots, S_{n-1}\right\}$ of $X$ such that $\tau S_{i}=S_{i+1}$

Received by the editors March 8, 1984.

1980 Mathematics Subject Classification. Primary 47A30, 28 D05.

(C) 1985 American Mathematical Society $0002-9939 / 85 \$ 1.00+\$ .25$ per page 
for $0 \leq i<n-1$. $S_{0}$ is called the base of $S$ (written $B(S)$ ), $S_{n-1}$ the top and $n$ the height.

2. Construction of $\tau$ and the main theorem. To illustrate the general method and simplify the computations we construct an ergodic automorphism $\tau$ which admits simple approximations with multiplicity 3 and has maximal spectral multiplicity equal to 2 . The general construction for $M$ a natural number is then a straightforward generalization of this.

$\tau$ will be defined on $(X, \mathfrak{B}, \mu)$, where $X=[0,1), \mathfrak{B}$ is the $\sigma$-algebra of Borel sets and $\mu$ is the Lebesgue measure. We proceed by the stacking method using three stacks. At the $n$th stage we will have three $\tau$-stacks $S_{n}^{0}, S_{n}^{1}$ and $S_{n}^{2}$ whose levels are intervals of length $1 / 3^{n+1}$ and $\tau$ will map each level in $S_{n}^{i}$ except the top one linearly onto the level directly above. $\tau$ will be undefined on the top of $S_{n}^{i}$.

We define $S_{n}^{i}$ inductively, first putting $S_{0}^{0}=\left[0, \frac{1}{3}\right), S_{0}^{1}=\left[\frac{1}{3}, \frac{2}{3}\right)$ and $S_{0}^{2}=\left[\frac{2}{3}, 1\right)$. Suppose $S_{n}^{0}, S_{n}^{1}$ and $S_{n}^{2}$ have been constructed and have height $3^{n}$. Cut $B\left(S_{n}^{i}\right)$ into three intervals $I_{n}^{i}, J_{n}^{i}$ and $K_{n}^{i}$ of equal length and let $\tau$ map $\tau^{3^{n}-1} I_{n}^{i}$ linearly onto $K_{n}^{i+1}$ and $\tau^{3^{n}-1} K_{n}^{i}$ linearly onto $J_{n}^{i+2}(i=0,1,2$; where $i+1, i+2$ are interpreted $\bmod 3)$.

This defines $\tau$-stacks $S_{n+1}^{0}, S_{n+1}^{1}, S_{n+1}^{2}$ of height $3^{n+1}$ and bases $I_{n}^{0}, I_{n}^{1}, I_{n}^{2}$, respectively. Throughout we shall denote the base of $S_{n}^{i}$ by $B_{n}^{i}$, i.e. $B\left(S_{n}^{i}\right)=B_{n}^{i}$, $i=0,1,2$.

We now state the main theorem. The major part of the proof follows from a number of propositions given in the next section.

THEOREM 1. $\tau$ is an ergodic automorphism with maximal spectral multiplicity equal to two.

PROOF. Regarding $\tau$ as a single stack with base $\bigcup_{i=0}^{2} B_{n}^{i}$ and height $3^{n}$, the ergodicity follows from the usual arguments for single stacks (see Friedman [4]). Note also that $\tau$ has as eigenvalues all the $3^{n}$ th roots of unity. The rest of the proof follows from Propositions 1, 2 and 3 of $\S 3$.

3. Invariant subspaces for $U_{\tau}$ and the proof of the theorem. We introduce invariant subspaces $H_{k}, k=0,1,2$, in $L^{2}(X)$ by first defining $\sigma: X \rightarrow X$ by $\sigma(x)=x+\frac{1}{3}(\bmod 1)$ and writing $H_{k}=\left\{f \in L^{2}(X): U_{\sigma} f=\omega^{k} f\right\}, k=0,1,2$; where $\omega=e^{2 \pi i / 3}$ and $U_{\sigma}: L^{2}(X) \rightarrow L^{2}(X)$ is the operator $\left(U_{\sigma} f\right)(x)=f\left(\sigma^{-1} x\right)$.

LEMMA 1. (i) $\sigma$ and $\tau$ commute.

(ii) $H_{k}, k=0,1,2$, is invariant under $U_{\tau}$ and $L^{2}(X)=H_{0} \oplus H_{1} \oplus H_{2}$.

Proof. (i) By induction we see that $\sigma\left(\tau^{k} B_{n}^{0}\right)=\tau^{k}\left(B_{n}^{1}\right), \sigma\left(\tau^{k} B_{n}^{1}\right)=B_{n}^{2}$ and $\sigma\left(\tau^{k} B_{n}^{2}\right)=B_{n}^{0}$ for $k=0,1, \ldots, 3^{n}-1 ; n=1,2, \ldots$ It follows that $\tau$ and $\sigma$ commute.

(ii) From (i) $H_{k}$ is invariant under $U_{\tau}, k=0,1,2$. Furthermore $L^{2}(X)$ has an orthonormal basis $\left\{\phi_{n}: n \in \mathbf{Z}\right\}$, where $\phi_{n}(z)=z^{n}, z \in K$ (identifying $X$ with the unit circle $K$ in the complex plane). Since $\phi_{3 m+k} \in H_{k}$ for $m \in \mathbf{Z}, k=0,1,2$, it follows that $L^{2}(X)=H_{0} \oplus H_{1} \oplus H_{2}$.

PROPOSITION 1. $U_{\tau} \mid H_{0}$ has discrete spectrum with eigenvalues precisely all the $3^{n}$ th roots of unity. 
ProOF. Denote $\mathfrak{B}_{\zeta}=\{E: \sigma E=E\}$ as the sub- $\sigma$-algebra of $\mathfrak{B}$ of sets invariant under $\sigma$, and let $\zeta$ be the corresponding partition of $X$. Note that $H_{0}=L^{2}(X / \zeta)$ and the conditional expectation $E(\cdot \mid \varsigma): L^{2}(X) \rightarrow L^{2}(X / \varsigma)$ is the projection onto $H_{0}$. Using these considerations it can be seen that $U_{\tau} \mid H_{0}$ arises from the von NeumannKakutani type transformation obtained by cutting $[0,1)$ into three intervals of equal length, stacking into a single column and repeating the process to obtain a column of height $3^{n}$ at the $n$th stage. (In face, $\tau$ acting on the $\sigma$-algebra $\mathfrak{B}_{\zeta}$ is precisely this transformation.) In particular, the eigenvalues are precisely all the $3^{n}$ th roots of unity; each eigenvalue is simple so $U_{\tau} \mid H_{0}$ has simple spectrum.

Proposition 2. $U_{\tau} \mid H_{1}$ and $U_{\tau} \mid H_{2}$ each have simple continuous spectrum.

ProOF. (i) For the continuity of spectrum of $U_{\tau} \mid H_{1}$, let $f \in H_{1}$ and $\lambda \in K$ with $U_{\tau} f=\lambda f$. Then $U_{\sigma} f=\omega f$ so $U_{\sigma} f^{3}=f^{3}$, or $f^{3} \in H_{0}$. Now $U_{\tau} f^{3}=\lambda^{3} f^{3}$ and since $U_{\tau} \mid H_{0}$ only has eigenvalues which are $3^{n}$ th roots of unity, $\lambda^{3}$ is a $3^{n}$ th root of unity and so also is $\lambda$. It follows that $U_{\tau}$ has two orthogonal eigenfunctions corresponding to the same eigenvalue, contradicting the ergodicity of $\tau$. A similar argument applies to $U_{\tau} \mid H_{2}$.

(ii) To see that $U_{\tau} \mid H_{2}$ has simple spectrum write

$$
f_{n}=\chi_{B_{n}^{0}}+\omega \chi_{\sigma B_{n}^{0}}+\omega^{2} \chi_{\sigma^{2} B_{n}^{0}}=\chi_{B_{n}^{0}}+\omega \chi_{B_{n}^{1}}+\omega^{2} \chi_{B_{n}^{2}} .
$$

Then

$$
U_{\sigma} f_{n}=\chi_{\sigma B_{n}^{0}}+\omega \chi_{\sigma^{2} B_{n}^{0}}+\omega^{2} \chi_{\sigma^{3} B_{n}^{0}}=\omega^{2} f_{n}
$$

so that $f_{n} \in H_{2}$. Clearly $\left\|f_{n}\right\| \rightarrow 0$ as $n \rightarrow \infty$. Let $Z\left(f_{n}\right)$ denote the cyclic subspace generated by $f_{n}$; then we claim $Z\left(f_{n}\right) \nearrow H_{2}$ as $n \rightarrow \infty$. It follows from Baxter [1, Lemma 14] that $U_{\tau} \mid H_{2}$ has simple spectrum. A similar argument using $g_{n}=\chi_{B_{n}^{0}}+\omega^{2} \chi_{B_{n}^{1}}+\omega \chi_{B_{n}^{2}}$ implies $U_{\tau} \mid H_{1}$ has simple spectrum.

To show that $Z\left(f_{n}\right) \nearrow H_{2}$ let $f \in L^{2}(X)$ and notice that the projection $E: L^{2}(X) \rightarrow H_{2}$ is defined by $E(f)=\frac{1}{3}\left(f+\omega U_{\sigma} f+\omega^{2} U_{\sigma^{2}} f\right)$. It is sufficient to show that $E(f)$ can be approximated arbitrarily closely by linear combinations of $U_{\tau}^{m}\left(f_{n}\right), m \in \mathbf{Z}$. Since the partition $\left\{\tau^{j} B_{n}^{i}: i=0,1,2 ; j=0,1, \ldots, 3^{n}-1 ; n=\right.$ $0,1, \ldots\}$ generates the Borel sets, there exists

$$
h_{n}=\sum_{i=0}^{2} \sum_{j=0}^{3^{n}-1} a_{i j} \chi_{\tau^{j} B_{n}^{i}}, \quad a_{i j} \in \mathbf{C}, n \in \mathbf{N},
$$

satisfying $\left\|f-h_{n}\right\|<\epsilon$ or $\left\|E(f)-E\left(h_{n}\right)\right\|<\epsilon$. Now $E\left(h_{n}\right) \in Z\left(f_{n}\right)$ since

$$
\begin{aligned}
E\left(h_{n}\right) & =\sum_{i=0}^{2} \sum_{j=0}^{3^{n}-1} a_{i j} \frac{1}{3}\left(\chi_{\tau^{j} B_{n}^{i}}+\omega \chi_{\tau^{j} \sigma B_{n}^{i}}+\omega^{2} \chi_{\tau^{j} \sigma^{2} B_{n}^{i}}\right) \\
& =\frac{1}{3} \sum_{i=0}^{2} \sum_{j=0}^{3^{n}-1} a_{i j} U_{\tau}^{j}\left(\chi_{B_{n}^{i}}+\omega \chi_{\sigma B_{n}^{i}}+\omega^{2} \chi_{\sigma^{2} B_{n}^{i}}\right) \\
& =\frac{1}{3} \sum_{i=0}^{2} \sum_{j=0}^{3^{n}-1} \omega^{-i} a_{i j} U_{\tau}^{j}\left(f_{n}\right) \in Z\left(f_{n}\right),
\end{aligned}
$$

and the result follows. 
PROPOSITION 3. $U_{\tau} \mid H_{1} \oplus H_{2}$ has maximal spectral multiplicity equal to two.

PROOF. $U_{\tau} \mid H_{i}, i=1,2$, each have simple continuous spectrum, so the multiplicity of $U_{\tau} \mid H_{1} \oplus H_{2}$ cannot exceed two. Suppose $U_{\tau} \mid H_{1} \oplus H_{2}$ has multiplicity equal to one. Then there exists $w \in H_{1} \oplus H_{2}$ with $Z(w)=H_{1} \oplus H_{2}$. If $f_{n}$ and $g_{n}$ are as defined in Proposition $2, f_{n}, g_{n} \in Z(w)$ and $Z\left(f_{n}\right) \perp Z\left(g_{n}\right)$. Denote the maximal spectral types of $U_{\tau} \mid Z\left(g_{n}\right)$ and $U_{\tau} \mid Z\left(f_{n}\right)$ by $\rho_{1}^{n}$ and $\rho_{2}^{n}$, respectively. Then $\rho_{1}^{n}$ and $\rho_{2}^{n}$ are mutually singular (see Parry $[6$, p. 93]). The theorem will follow if we show $\rho_{1}^{n}=\rho_{2}^{n}, n \in \mathbf{N}$, and for this it suffices to show $\left(U_{\tau}^{m} f_{n}, f_{n}\right)=\left(U_{\tau}^{m} g_{n}, g_{n}\right)$ for all $m \in \mathbf{Z}, n \in \mathbf{N}$.

Now

$$
\begin{aligned}
\left(U_{\tau}^{m} f_{n}, f_{n}\right) & =\int U_{\tau}^{m} f_{n} \cdot \bar{f}_{n} d \mu \\
& =\int\left(\chi_{\tau^{m} B_{n}^{0}}+\omega \chi_{\tau^{m} \sigma B_{n}^{0}}+\omega^{2} \chi_{\tau^{m} \sigma^{2} B_{n}^{0}}\right)\left(\chi_{B_{n}^{0}}+\omega^{2} \chi_{\sigma B_{n}^{0}}+\omega \chi_{\sigma^{2} B_{n}^{0}}\right) d \mu \\
& =3 \mu\left(\tau^{m} B_{n}^{0} \cap B_{n}^{0}\right)+3 \omega^{2} \mu\left(\tau^{m} B_{n}^{0} \cap \sigma B_{n}^{0}\right)+3 \omega \mu\left(\tau^{m} B_{n}^{0} \cap \sigma^{2} B_{n}^{0}\right)
\end{aligned}
$$

since $\sigma$ is measure preserving. A similar calculation shows that

$$
\left(U_{\tau}^{m} g_{n}, g_{n}\right)=3 \mu\left(\tau^{m} B_{n}^{0} \cap B_{n}^{0}\right)+3 \omega \mu\left(\tau^{m} B_{n}^{0} \cap \sigma B_{n}^{0}\right)+3 \omega^{2} \mu\left(\tau^{m} B_{n}^{0} \cap \sigma^{2} B_{n}^{0}\right)
$$

for all $m \in \mathbf{Z}, n \in \mathbf{N}$. Thus the result follows on noting that $\mu\left(\tau^{m} B_{n}^{0} \cap \sigma B_{n}^{0}\right)=$ $\mu\left(\tau^{m} B_{n}^{0} \cap \sigma^{2} B_{n}^{0}\right)$ for all $m \in \mathbf{Z}, n \in \mathbf{N}$, as can be seen from the symmetry of the construction of $\tau$.

\section{REFERENCES}

1. J. R. Baxter, A class of ergodic transformations having simple spectrum, Proc. Amer. Math. Soc. 27 (1971), 275-279.

2. R. V. Chacon, Approximation and spectral multiplicity, Lecture Notes in Math., Vol. 160, SpringerVerlag, 1970, pp. 18-27.

3. A. Del Junco, A transformation with simple spectrum which is not rank one, Canad. J. Math. 29 (1977), 655-663.

4. N. A. Friedman, Introduction to ergodic theory, Van Nostrand, Princeton, N. J., 1970.

5. V. I. Oseledec, The spectrum of ergodic automorphisms, Dokl. Akad. Nauk SSSR 168 (1966), 776-779. (Russian)

6. W. Parry, Topics in ergodic theory, Cambridge Univ. Press, New York, 1981.

7. E. A. Robinson, Jr., Ergodic measure preserving transformations with arbitrary finite spectral multiplicities, Invent. Math. 72 (1983), 299-314.

Department of mathematics, University of Cape Town, Rondebosch, 7700 C. P., SOUTH AFrICA 